\title{
Factores docentes y su asociación con el índice de reprobación en alumnos de nivel superior
}

MNC. Alejandra Karina Pérez Jaimes, Mexicana.

ORCID https://orcid.org/0000-0003-3079-1782

coord.nutricion@colmexuni.edu.mx

MCS. César Estrada Uziel Estrada Reye, Mexicano.

ORCID https://orcid.org/ 0000-0003-4932-2214

cesar.estrada.lic@colmexuni.edu.mx

Campus Licenciaturas

Grupo Colegio Mexiquense

Dr. César Enrique Estrada Gutiérrez, Mexicana.

ORCID https://orcid.org/ 0000-0002-0936-8157

cestradag@uaemex.mx

Facultad de Contaduría y Administración

Universidad Autónoma del Estado de México

Recibido: 14 de agosto del 2020

Aceptado: 31 de enero del 2021

\section{Resumen}

Existen diferentes variables que influyen en el índice de reprobación considerando los factores del alumno y docente; actualmente la presión social que surge por definir a los docentes de buena calidad implica el estudio de diferentes factores. En diversas ocasiones el docente opta por sistemas de evaluación que valoran el desempeño de los alumnos como indicador, el presente artículo refiere diferentes variables que involucran al docente en relación al resultado del alumno. Asociar de los factores docentes y el Índice de Reprobación en alumnos de Nivel Superior. El tipo de investigación fue analítico, observacional, prospectivo y transversal. Se utilizó una técnica cuantitativa, estudiadas con medidas de tendencia central (media) y de dispersión (rangos mínimos, máximos y DE), la relación de los factores docentes y el índice de reprobación fue la prueba estadística de $t$ de student. La clasificación de Ciencias mostro un predominio del $36.7 \%$ $(n=33)$ para la Ciencias Sociales, el 27.8\% $(n=25)$ respecto a las Ciencias Exactas, un $35.6 \%(n=32)$ las Ciencias de la Salud, para la relación del índice de reprobación con el nivel máximo de estudios y evaluación de coordinación, se realizó la prueba estadística de chi-cuadrada, obteniendo un valor de p de 0.02 , siendo este un valor estadísticamente significativo. La formación docente permanente es una necesidad. Las demandas del 
desarrollo y la transformación social exigen un cambio en su concepción que requiere un enfoque que priorice al docente como agente activo de su aprendizaje, desde las potencialidades de su desarrollo, con carácter autotransformador y transformador de la realidad social.

Palabras clave: aprendizaje, reprobación y preparación docente

\title{
"Teaching factors and their association with the failure index in higher education students"
}

\begin{abstract}
There are different variables that influence the index of failure considering the factors of the student and teacher; currently the social pressure that arises to define teachers of good quality impedes the study of different factors. In several occasions the teacher opts for evaluation systems that value the performance of the students as an indicator, the present article refers to different variables that involve the teacher in relation to the student's result. To associate of the teaching factors and the Index of Failure in students of Higher Level. The type of research was analytical, obsevacional, prospective and transversal. A quantitative technique was used, studied with measures of central tendency (mean) and dispersion (minimum ranges, maximum and SD ranges), the ratio of teaching factors and the index of failure was the statistical test of student t. Results: the Sciences classification showed a predominance of $36.7 \%(n=33)$ for the Social Sciences, $27.8 \%(n=25)$ for the Exact Sciences, 35.6\% $(n=32)$ for the Health Sciences, for the relation of the reprobation index with the maximum level of studies and coordination evaluation, the chi-square statistical test was performed, obtaining a $\mathrm{p}$ value of 0.02 , this being a statistically significant value. Continuing teacher education is a necessity. The demands of development and social transformation require a change in its conception that requires an approach that prioritizes the teacher as an active agent of his learning, from the potentialities of his development, with an autotransforming and transforming character of social reality.
\end{abstract}

Keywords: learning, reprobation and teacher preparation 


\section{Introducción}

La necesidad de evaluar el desempeño en la Educación Superior es una realidad reconocida desde hace mucho tiempo en numerosos países. Tradicionalmente, esta labor ha sido realizada según tres perspectivas: la evaluación de las instituciones dentro de un país; la evaluación de los departamentos de la institución, y la evaluación de las titulaciones y de los cursos o de los programas de Educación Superior (Moreira\&Santos, 2016).

La formación del docente debe ser de forma permanente y continua, como reconocen los autores, para que este sea un verdadero agente transformador de la sociedad. Se requiere de nuevas visiones de sus aspectos más generales conceptuales en el contexto actual, donde los saberes pragmáticos, instrumentalistas, tecnológicos soslayan el desarrollo humano del docente en un proceso de significación personal y social (Nieva\&Martínez, 2016).

La participación del docente en la educación es considerado hasta cierto punto el conclave de la educación teniendo como prerrogativas lo siguiente ¿Cuál es el factor que no permite el correcto desarrollo de la enseánza aprendizaje? ¿La preparación profesional o pedagógica será pieza clave? ¿Cuál es el factor que más se debe cuidar em la práctica de la docencia?

La idea básica es a primera vista indiscutible: un buen docente es aquél que logra que sus alumnos aprendan; y, si los sistemas educativos cuentan con información comparable y confiable sobre el rendimiento de los alumnos, entonces no es necesario observar el trabajo de cada maestro para valorar su propio desempeño, sino que bastará con inferirlo a partir del rendimiento de sus alumnos (Martínez Rizo, 2011).

\section{Antecedentes}

James, Baldwin y McInnis (1999) mencionan que los factores que influyen en la elección que hacen los alumnos de una determinada institución tienen que ver con cuestiones de calidad; es decir, que este considera que el docente presentara la misma característica. Por lo anterior, el principal objetivo de la institución educativa será seleccionar docentes con diferentes características todas de índole competitiva. 
En otra investigación se mostró que la calidad de la docencia impartida es el factor principal que los estudiantes tienen en cuenta a la hora de elegir una universidad, hasta el punto de superar otros factores, como son el de las instalaciones y recursos académicos disponibles, o incluso las perspectivas de empleabilidad (Shah y Nair, 2010)

En la actualidad han generado diferentes planteamientos sobre los modelos educativos; ello supone una alternativa a los modelos tradicionales, al no aceptar a nivel formal el currículo escolar como algo cerrado, sino intentando cambiarlo para tratar de responder a los retos de la inclusión (Escarvajal, 2010). En donde, no se considera como variable a modificar la preparación del docente sea de tipo pedagógica o profesionalizaste.

Una de los principales planteamientos ha sido un enfoque inclusivo que es, ante todo, una cuestión "que se mueve en el terreno de las concepciones básicas y de las actitudes o disposición ante la respuesta educativa que ha de darse a la diversidad, más que en el terreno de unas determinadas acciones concretas" (Sánchez Palomino y Lázaro, 2011, p. 90).

La experiencia profesional está representada con mayor validez entre las finalidades de la institución universitaria destacan, además de proporcionar una preparación técnica y profesional adecuada, contribuir a la formación de personas maduras, reflexivas y críticas, y despertar en el alumno universitario el interés por cuestiones cívicas (Llano, 2003).

La preparación pedagógica en el docente de diferentes disciplinas refiere una organización previa en clase que se verá reflejada en el aprendizaje del alumno; desde esta perspectiva, para Martin-Kniep (2001) el portafolio es “un instrumento de evaluación del desempeño docente, que consiste en una colección de evidencias de los procesos de enseñanza y reflexión de la propia práctica en función de estándares establecidos” (p.17).

El rendimiento académico ha sido estudiado por infinidad de personajes que, en su mayoría, definen como el resultado cuantitativo obtenido durante el proceso de aprendizaje conforme a las evaluaciones que realiza el docente mediante pruebas objetivas y otras actividades complementarias. Bricklin y Bricklin (1988), aluden a un factor del que no es responsable el estudiante, es decir, los prejuicios que el docente puede llegar a tener con respecto a sus alumnos y por los cuales nunca debe dejarse llevar. El 
grado de cooperación de los alumnos influye en la forma como el maestro los considera. Si este piensa que son más inteligentes o mejores estudiantes, afecta su rendimiento escolar. Jiménez (2000), entiende como rendimiento escolar el nivel de conocimientos demostrados en un área o materia comparado con la norma de edad y nivel académico.

La responsabilidad en cuanto al rendimiento del alumno la tiene el docente, así como el proceso de enseñanza-aprendizaje, la metodología del profesor, el alumno en sí, el apoyo familiar, la situación social, etcétera. Se entiende que el rendimiento no son solo las calificaciones del estudiante, sino también sus habilidades, destrezas, aptitudes, ideales, intereses, etcétera. (Figueroa, 2004) Sin embargo, algo es contundente en este panorama: si falla alguna de las características mencionadas, indudablemente tendremos a un alumno reprobado. El objetivo fue asociar de los factores docentes y el Índice de Reprobación en alumnos de Nivel Superior

El presente trabajo fue expuesto a Dirección (de la universidad evaluada) quien autorizó el uso de la base datos de los docentes y alumnos, siendo beneficiados por la identificación de áreas de mejora. Los datos fueron recolectados con ayuda del departamento de Dirección y Pedagogía de la universidad. El análisis de los datos se realizó con la paquetería estadística SPSS Versión 23, posterior de la interpretación se comparó con otras investigaciones.

\section{Metodología}

La presente investigación fue de tipo analítico, observacional, prospectivo y transversal. La población son docentes pertenecientes al Nivel Superior de la Universidad estudiada. El tipo de muestreo será no probabilístico a conveniencia, ya que se trabajará con 90 docentes de diferentes clasificaciones (Salud, Exacta y Sociales).

Para la presente investigación se utilizó una técnica cuantitativa, estudiadas con medidas de tendencia central (media) y de dispersión (rangos mínimos, máximos y DE), en cuanto a las variables cualitativas los resultados se generaron con frecuencias y porcentajes. La relación de los factores docentes y el índice de reprobación, fue la prueba estadística de $t$ de student.

Todos los datos se analizaron a través del paquete estadístico SPSS versión 25, 
tomando como significativo un p valor menor a 0.05 .

\section{Resultados}

Tabla 1. Clasificación de Ciencias

\begin{tabular}{ccc}
\hline & Frecuencia & Porcentaje \\
\hline Ciencias de la Salud & 32 & 35.6 \\
Ciencias Exactas & 25 & 27.8 \\
Ciencias Sociales & 33 & 36.7 \\
\hline
\end{tabular}

En la tabla 1. Clasificación de Ciencias mostro un predominio del 36.7\% $(n=33)$ para la Ciencias Sociales, el $27.8 \%(n=25)$ respecto a las Ciencias Exactas y un $35.6 \%(n=32)$ las Ciencias de la Salud.

Tabla 2. Nivel máximo de estudios

\begin{tabular}{ccc}
\hline & Frecuencia & Porcentaje \\
\hline Licenciatura & 55 & 61.1 \\
Maestría & 32 & 35.6 \\
Doctorado & 3 & 3.3 \\
\hline
\end{tabular}

En la tabla 2. Nivel máximo de estudios, muestra un $61.1 \%(n=55)$ de docentes que cuentan con licenciatura, seguido de un $25.6 \%(n=32)$ para maestría, y solamente el $3.3 \%$ $(n=3)$ con doctorado.

Tabla 3. Experiencia profesional docente

\begin{tabular}{ccc} 
& Frecuencia & Porcentaje \\
\hline$<2$ años & 62 & 68.9 \\
2 a 5 años & 21 & 23.3 \\
$>$ > años & 7 & 7.8 \\
\hline
\end{tabular}

En cuanto a la experiencia profesional docente, el $68.9 \%(n=62)$ presentó una experiencia menor a 2 años, el $23.3 \%(n=21)$ de 2 a 5 años y solo un $7.8 \%(n=7)$ mayor a 5 años. 
Tabla 4. Preparación pedagógica

\begin{tabular}{ccc}
\hline & Frecuencia & Porcentaje \\
\hline Ningún curso & 49 & 54.4 \\
1 a 3 cursos & 37 & 41.1 \\
$>$ 4 cursos & 4 & 4.4 \\
\hline
\end{tabular}

La preparación pedagógica mayor a 4 cursos fue del 4.4\% (n=4), el 41.1\% (n=37) presentó de 1 a 3 cursos y el 54.4\% (n=49) ningún curso de preparación pedagógica.

Tabla 5. Evaluación por coordinación

\begin{tabular}{ccc}
\hline & Frecuencia & Porcentaje \\
\hline Excelente & 2 & 2.2 \\
Bueno & 72 & 80.0 \\
Regular & 12 & 13.3 \\
Bajo & 4 & 4.4 \\
\hline
\end{tabular}

La tabla 5. Evaluación por coordinación, obtuvo un $80 \%(\mathrm{n}=72)$ de un desempeño bueno, el $13.3 \%(n=12)$ de regular, un $4.4 \%(n=4)$ en bajo y solo un $2.2 \%(n=2)$ en excelente.

Tabla 6. Evaluación por área pedagógica

\begin{tabular}{ccc}
\hline & Frecuencia & Porcentaje \\
\hline Excelente & 4 & 4.4 \\
Bueno & 75 & 83.3 \\
Regular & 4 & 4.4 \\
Bajo & 7 & 7.8 \\
\hline
\end{tabular}

La evaluación de la plantilla docente por el área pedagógica mostró un predominio del $83.3 \%(n=75)$ en bueno, seguido por el $7.8 \%(n=7)$ en bajo y un $4.4 \%(n=4)$ en regular y excelente.

\begin{tabular}{ccc}
$\begin{array}{c}\text { Tabla 7. Índice de reprobación } \\
\text { Porcentaje de Índice de } \\
\text { Reprobación }\end{array}$ & Frecuencia & Porcentaje \\
\hline$<\mathbf{1 0}$ & 65 & 72.2 \\
$\mathbf{1 1 - 2 0}$ & 7 & 7.8 \\
$\mathbf{2 1 - 4 0}$ & 11 & 12.2 \\
$\mathbf{4 1 - 5 0}$ & 3 & 3.3 \\
$>\mathbf{5 0}$ & 4 & 4.4 \\
\hline
\end{tabular}


El índice de reprobación menor al 10\% fue de un 72.2\% (n=65), seguido por un rango del 21 al $40 \%$ con un $12.2 \%(n=11)$, el $7.8 \%(n=7)$ en un rango de 11 al 20\%, un $4.4 \%(n=4)$ mayor al 50\% de reprobación y el 3.3\% (n=3) de 41 al $50 \%$.

Tabla 8. Relación del índice de reprobación con la clasificación en ciencias

\begin{tabular}{cccccccccccc}
\hline & $<10$ & \multicolumn{3}{c}{$11-20$} & \multicolumn{2}{c}{$21-40$} & $41-50$ & $>50$ & \multicolumn{1}{c}{} \\
\hline & Frec & $\%$ & Frec & $\%$ & Frec & $\%$ & Frec & $\%$ & Frec & $\%$ & \\
$\begin{array}{c}\text { Ciencias de } \\
\text { la Salud }\end{array}$ & 23 & 35.4 & 3 & 42.9 & 4 & 36.4 & 1 & 33.3 & 1 & 25 & \\
$\begin{array}{c}\text { Ciencias } \\
\text { Exactas }\end{array}$ & 18 & 27.7 & 1 & 14.3 & 3 & 27.3 & 1 & 33.3 & 2 & 50 & 0.99 \\
$\begin{array}{c}\text { Ciencias } \\
\text { Sociales }\end{array}$ & 24 & 36.9 & 3 & 42.9 & 4 & 36.4 & 1 & 33.3 & 1 & 25 & \\
\hline
\end{tabular}

Para la relación del índice de reprobación con la clasificación en ciencias, se realizó la prueba estadística de chi-cuadrada, obteniendo un valor de p de 0.99 , siendo este un valor estadísticamente no significativo.

Tabla 9. Relación del índice de reprobación con el nivel máximo de estudios \% Índice de Reprobación

\begin{tabular}{|c|c|c|c|c|c|c|c|}
\hline & & $<10$ & $11-20$ & $21-40$ & $41-50$ & $>50$ & $\mathrm{p}$ \\
\hline \multirow[t]{2}{*}{ Licenciatura } & Recuento & 42 & 4 & 7 & 0 & 2 & \\
\hline & $\begin{array}{l}\% \text { dentro de } \\
\text { IND_DE_REPR }\end{array}$ & $64.6 \%$ & $57.1 \%$ & $63.6 \%$ & $0.0 \%$ & $50.0 \%$ & \\
\hline \multirow[t]{2}{*}{ Maestría } & Recuento & 16 & 1 & 3 & 2 & 1 & \\
\hline & $\begin{array}{l}\text { \% dentro de } \\
\text { IND_DE_REPR }\end{array}$ & $24.6 \%$ & $14.3 \%$ & $27.3 \%$ & $66.7 \%$ & $25.0 \%$ & 0.02 \\
\hline \multirow{2}{*}{ Doctorado } & Recuento & 0 & 1 & 0 & 1 & 1 & \\
\hline & $\begin{array}{l}\% \text { dentro de } \\
\text { IND_DE_REPR }\end{array}$ & $0.0 \%$ & $14.3 \%$ & $0.0 \%$ & $33.3 \%$ & $25.0 \%$ & \\
\hline
\end{tabular}

Para la relación del índice de reprobación con el nivel máximo de estudios, se realizó la prueba estadística de chi-cuadrada, obteniendo un valor de p de 0.02 , siendo este un valor estadísticamente significativo. 
Tabla 10. Relación del índice de reprobación con la experiencia profesional \% Índice de Reprobación

\begin{tabular}{llrrrrrr} 
& \multicolumn{1}{c}{$<10$} & $11-20$ & $21-40$ & $41-50$ & $>50$ & $\mathrm{p}$ \\
\hline \multirow{3}{*}{$<$ 2 años } & Recuento & 45 & 4 & 6 & 3 & 4 & \\
& \% dentro de & $69.2 \%$ & $57.1 \%$ & $54.5 \%$ & $100.0 \%$ & $100.0 \%$ & \\
& IND_DE_REPR & & & & & & \\
& Recuento a 5 años & 14 & 2 & 5 & 0 & 0 & 0.48 \\
& $\%$ dentro de & $21.5 \%$ & $28.6 \%$ & $45.5 \%$ & $0.0 \%$ & $0.0 \%$ & \\
& IND_DE_REPR & & & & & & \\
& Recuento & 6 & 1 & 0 & 0 & 0 & \\
& \% años dentro de & $9.2 \%$ & $14.3 \%$ & $0.0 \%$ & $0.0 \%$ & $0.0 \%$ & \\
& IND_DE_REPR & & & & & &
\end{tabular}

Para la relación del índice de reprobación con la experiencia profesional, se realizó la prueba estadística de chi-cuadrada, obteniendo un valor de p de 0.48 , siendo este un valor estadísticamente no significativo.

Tabla 13. Relación del índice de reprobación con la preparación pedagógica

IND_DE_REPR

\begin{tabular}{|c|c|c|c|c|c|c|c|c|}
\hline & & & $<10$ & $11-20$ & $21-40$ & $41-50$ & $>\mathbf{5 0}$ & p \\
\hline \multirow{6}{*}{ P_PEDAGOGO } & \multirow{2}{*}{$\begin{array}{l}\text { Ningun } \\
\text { curso }\end{array}$} & Recuento & 39 & 3 & 4 & 2 & 1 & 0.33 \\
\hline & & $\begin{array}{l}\text { \% dentro de } \\
\text { IND_DE_REPR }\end{array}$ & $60.0 \%$ & $42.9 \%$ & $36.4 \%$ & $66.7 \%$ & $25.0 \%$ & \\
\hline & \multirow{2}{*}{$\begin{array}{l}1 \text { a } 3 \\
\text { cursos }\end{array}$} & Recuento & 23 & 4 & 7 & 1 & 2 & 0.33 \\
\hline & & $\begin{array}{l}\text { \% dentro de } \\
\text { IND_DE_REPR }\end{array}$ & $35.4 \%$ & $57.1 \%$ & $63.6 \%$ & $33.3 \%$ & $50.0 \%$ & \\
\hline & \multirow{2}{*}{$\begin{array}{l}>4 \\
\text { cursos }\end{array}$} & Recuento & 3 & 0 & 0 & 0 & 1 & 0.33 \\
\hline & & $\begin{array}{l}\text { \% dentro de } \\
\text { IND_DE_REPR }\end{array}$ & $4.6 \%$ & $0.0 \%$ & $0.0 \%$ & $0.0 \%$ & $25.0 \%$ & \\
\hline
\end{tabular}

Para la relación del índice de reprobación con la preparación pedagógica, se realizó la prueba estadística de chi-cuadrada, obteniendo un valor de p de 0.33 , siendo este un valor estadísticamente no significativo. 
Tabla 14. Relación del índice de reprobación con la evaluación de coordinación

IND_DE_REPR

\begin{tabular}{|c|c|c|c|c|c|c|c|c|}
\hline \multirow{2}{*}{ EVAL_COOR } & & & $<10$ & 11-20 & 21-40 & 41-50 & $>\mathbf{5 0}$ & $\mathbf{p}$ \\
\hline & Excelente & Recuento & 0 & 0 & 0 & 1 & 1 & 0.01 \\
\hline & & $\%$ dentro de & $0.0 \%$ & $0.0 \%$ & $0.0 \%$ & $33.3 \%$ & $25.0 \%$ & \\
\hline & & IND_DE_REPR & & & & & & \\
\hline & Bueno & Recuento & 51 & 7 & 9 & 2 & 3 & 0.01 \\
\hline & & $\%$ dentro de & $78.5 \%$ & $100.0 \%$ & $81.8 \%$ & $66.7 \%$ & $75.0 \%$ & \\
\hline & & IND_DE_REPR & & & & & & \\
\hline & Regular & Recuento & 10 & 0 & 2 & 0 & 0 & 0.01 \\
\hline & & $\%$ dentro de & $15.4 \%$ & $0.0 \%$ & $18.2 \%$ & $0.0 \%$ & $0.0 \%$ & \\
\hline & & IND_DE_REPR & & & & & & \\
\hline & Bajo & Recuento & 4 & 0 & 0 & 0 & 0 & 0.01 \\
\hline & & $\%$ dentro de & $6.2 \%$ & $0.0 \%$ & $0.0 \%$ & $0.0 \%$ & $0.0 \%$ & \\
\hline
\end{tabular}

IND_DE_REPR

Para la relación del índice de reprobación con la evaluación de coordinación, se realizó la prueba estadística de chi-cuadrada, obteniendo un valor de $\mathrm{p}$ de 0.01 , siendo este un valor estadísticamente significativo.

Tabla 15. Relación del índice de reprobación con la evaluación pedagógica

IND_DE_REPR

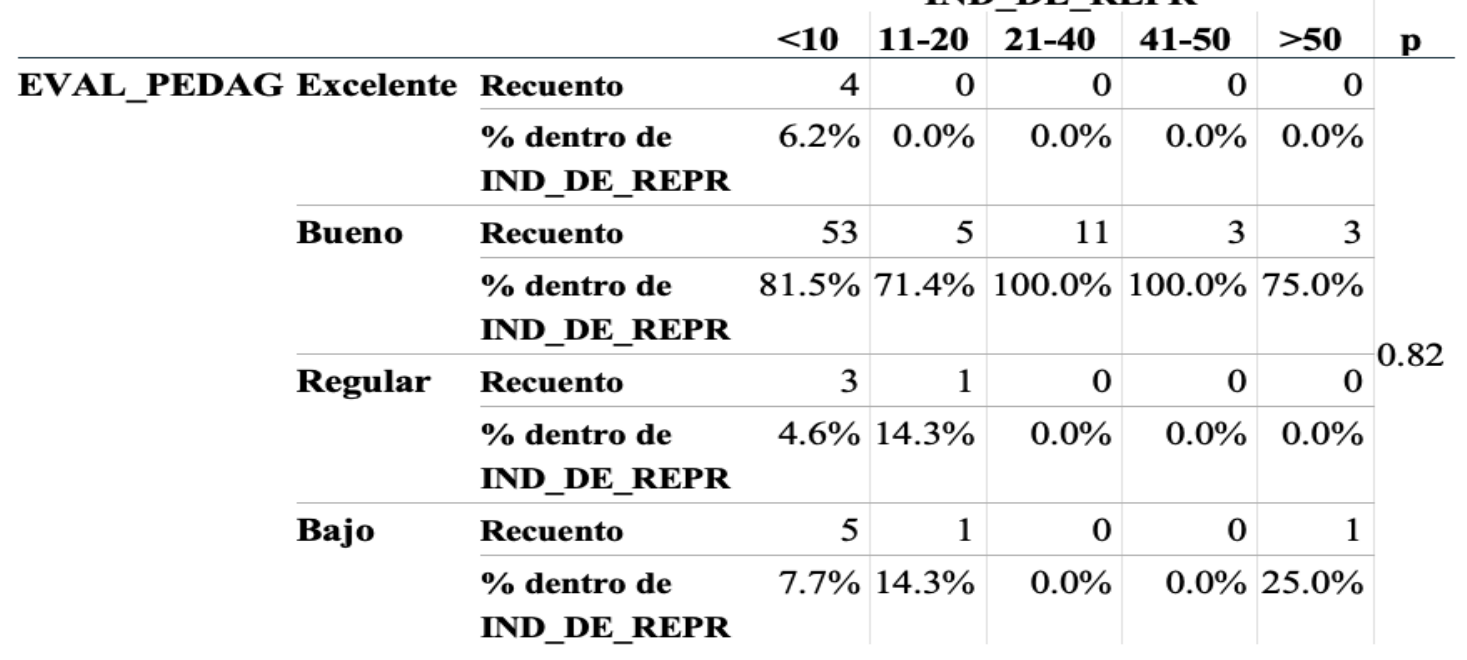

Para la relación del índice de reprobación con la evaluación pedagógica, se realizó la prueba estadística de chi-cuadrada, obteniendo un valor de $\mathrm{p}$ de 0.82 , siendo este un valor estadísticamente no significativo.

\section{Discusión}


La educación ha evidenciado su importancia en el desarrollo histórico de la sociedad, transmisora de la cultura que le ha antecedido en cada época o sistema social en particular. En ella el docente ha sido uno de los protagonistas principales en los procesos de desarrollo y transformación. Sin embargo, la formación docente no siempre ha recibido atención especial. Los estudios la abarcan desde diversos paradigmas, pero el docente como sujeto activo del aprendizaje ha sido escasamente atendido. Tanto desde la perspectiva de la formación inicial como permanente. El presente artículo abarca la formación permanente, la cual ha sido atendida por diversos autores (Freire, 2010; Martín, 2015; Delgado, 2013;Imbernón, 2011), que destacan la necesidad de cambiar el enfoque con el cual se realiza y comprende dicha formación. Al respecto se señala el papel protagónico y transformador de la realidad social que tiene el docente como educador

\section{Conclusiones}

La preparación profesional gener en el docente un mayor dominio del área profesionalizaste, combinado con su práctica profesional, la educación sintetiza la política, la cultura, la historia y el desarrollo de los seres humanos y la sociedad; la transmite y la transforma, donde el docente es un actor principal. La formación docente permanente es una necesidad. Las demandas del desarrollo y la transformación social exigen un cambio en su concepción que requiere un enfoque que priorice al docente como agente activo de su aprendizaje, desde las potencialidades de su desarrollo, con carácter autotransformador y transformador de la realidad social. La concepción del docente como aprendiz en el proceso de su formación abarca componentes y contenidos esenciales desde los histórico y cultural que propicia un aprendizaje significativo y desarrollador.

\section{Referencias bibliográficas}

Bricklin, M. \& Bricklin, B. (1988). Causas psicológicas del bajo. México, Paz-México.

Delgado B., Vanesa (2013). La formación del profesorado universitario. Análisis de los programas formativos de la Universidad de Burgos (2000-2011). Tesis Doctoral. Universidad de Burgos. Recuperado dehttps://dialnet.unirioja.es/servlet/articulo?codigo $=4491550$ 
Freire, P. (2010). Pedagogía de la autonomía y otros textos. La Habana: Caminos.

Escarvajal, A. (2010). La escuela inclusiva en una sociedad pluricultural y la importancia del trabajo colaborativo. Enseñanza \& Teaching, 28(2), 161-179.

Figueroa, C. (2004). Sistema de evaluaciones académicas (Primera ed.). El Salvador, Universitaria

Imbernón (2011). La formación pedagógica del docente universitario. Educação 36 (3).pp. 387-396.

Recuperado de http://www.nebrija.com/medios/encuentrosterceraclase/wpcontent/uploads/sites/8/2014/12/Ar

JAMES, R., BALDWIN, G. and MCINNIS, C., 1999. Which University?: The factors influencing the choices of prospective undergraduates. Department of Education, Training and Youth Affairs Canberra

Jiménez, M. (2000). Competencia social: intervención preventiva en la escuela. Infancia y Sociedad.

MARTÍN-KNIEP, G. (2001). Portfolios del desempeño de maestros, profesores y directivos. La sabiduría de la práctica. Buenos Aires: Paidós.

Moreira, L. M. y Santos, M. A. (2016). Evaluando la enseñanza en la Educación Superior: percepciones de docentes y discentes. Revista Electrónica de Investigación Educativa, 18(3), 19-36. Recuperado de http://redie.uabc.mx/redie/article/view/922

Nieva Chaves, José Antonio, \& Martínez Chacón, Orietta. (2016). Una nueva mirada sobre la formación docente. Revista Universidad y Sociedad, 8(4), 14-21. Recuperado en 30 de julio de 2020, de http://scielo.sld.cu/scielo.php?script=sci_arttext\&pid=S2218$36202016000400002 \& \operatorname{lng}=$ es\&tlng=es.

Llano, A. (2003). Repensar la universidad: la universidad ante lo nuevo. Madrid: Ediciones Internacionales Universitarias. 
Sánchez Palomino, A. y Lázaro, M. N. (2011). Hacia una perspectiva humanista de la Educación Especial. En Sánchez Palomino et al. (Eds.). Educación Especial y mundo digital (pp. 84-104). Universidad de Almería.

Shah, M. y Nair, C. S. (2010). Enrolling in higher education: The perceptions of stakeholders. Journal of Institutional Research, 15(1), 9-15. Recuperado de http://eric.ed.gov/?id=EJ1094125 